\title{
Intrapleural Fibrinolytic Therapy in Empyema Thoracis: Where are we now and where do we go from here?
}

\author{
Joseph L. Mathew ${ }^{1}$
}

Received: 15 October 2019 / Accepted: 16 October 2019/Published online: 11 November 2019

(C) Dr. K C Chaudhuri Foundation 2019

Intrapleural fibrinolytic therapy is often used in pediatric empyema, despite limitations with current evidence. There are few well-designed trials in children and most studies are (prospective or retrospective) observational reports with great diversity in the age of included children, type of fibrinolytic, timing of administration, number of doses, and outcomes evaluated. Naturally, systematic reviews also showed variable results. Nevertheless, the somewhat dated guidelines of the British Thoracic Society recommending fibrinolytics for empyema and complicated parapneumonic effusion [1] continue to be followed in that country [2]. The practice is followed in Australia as well [3]. However, some North American hospitals do not use fibrinolytics [4]. In fact, guidelines of the American Association of Thoracic Surgery do not emphasise intrapleural fibrinolytics at all [5]. Similarly the guidelines published by the Paediatric Infectious Diseases Society as well as Infectious Diseases Society of America, consider fibrinolytic as optional [6].

There are no pediatric guidelines in India. Medical units often use fibrinolytics, whereas Surgical units proceed with drainage and/or decortication. Our institution has been a pioneer in the systematic evaluation and usage of intrapleural streptokinase in empyema [7] and we recently confirmed its efficacy in loculated empyema, even when administered late i.e., beyond $14 \mathrm{~d}$ after disease onset (when it is not expected to work) [8]. Further, we found that 4-6 doses could be superior to the conventional 3 doses, although there was inadequate power to confirm this. We concluded that streptokinase administration is safe, efficacious, logistically feasible and affordable, reducing the difficulties and costs associated with surgical intervention. Hence we routinely use intrapleural streptokinase in empyema with septations, reserving surgical intervention only for children who fail to recover with 6 doses.

Joseph L. Mathew

dr.joseph.1.mathew@gmail.com

1 Department of Pediatric Pulmonology, Advanced Pediatrics Centre, PGIMER, Chandigarh, India
However, some institutions do not routinely use fibrinolytic therapy [Singhal K, personal communication], but the outcomes from such hospitals need evaluation.

This issue of the journal has a study evaluating the efficacy and safety of intrapleural urokinase in empyema [9]. The study was a retrospective record review over 4 consecutive years, comparing children who received 6 doses of intrapleural urokinase, $v s$. those who did not. Those receiving urokinase had shorter hospitalization (mean difference $6.8 \mathrm{~d}$, 95\% CI 3.1, $10.5 \mathrm{~d}$ ) and intercostal drainage duration (mean difference $1.8 \mathrm{~d}, 95 \% \mathrm{CI} 0.2,3.3 \mathrm{~d}$ ), although the proportion undergoing surgery was comparable in the two groups (odds ratio $0.49,95 \%$ CI $0.14,1.71)$. The authors concluded that intrapleural urokinase is a promising option.

What does this study [9] add to the evidence base? On the one hand, it re-confirms the efficacy and safety of intrapleural fibrinolytic therapy in childhood empyema. Further, to the best of my knowledge, this [9] is the first Indian study evaluating urokinase, although this has been used in several studies in developed countries. In the latter settings, urokinase, alteplase and tissue plasminogen activator are preferred over streptokinase because of the fear of adverse effects and immunological reactions with streptokinase.

Being a retrospective analysis of previously treated patients, this study [9] had the usual limitations of unstandardized definitions, treatment protocols, and data recording. The main flaw is that the basis for administering urokinase was not uniform in all patients. Children in an unspecified (earlier) period of the analysis did not receive it, whereas those admitted later were offered this. Further, those who could not afford urokinase did not receive any fibrinolytic (although they could/should have been offered the cheaper streptokinase). The impact of this selection bias is unclear, although the baseline characteristics of children in both groups was comparable. However, the volume of empyema at presentation (measured by surrogate markers such as width on chest X-ray or ultrasonography) was not described; this is important to assess comparability of the groups. 
The authors also did not describe the criteria for removal of chest tube drains, definition of treatment failure, criteria for surgical referral, time lag between referral and surgery, postsurgical morbidity \& hospitalization, etc. We have previously observed that children undergoing surgery have longer duration of hospitalization and intercostal drainage [8]. In this study, it is unclear whether the statistically significant and clinically impressive differences (in the mean durations of hospitalization and intercostal drainage) were because 9/44 $(20.5 \%)$ children in the non-urokinase group underwent surgery, compared to $4 / 10(10 \%)$ among those who received it. This conundrum can be resolved by comparing these outcomes in children who did not undergo surgery.

Children in this study had symptoms for 2-3 wk prior to presentation. Although it does not suggest that empyema was present for the same duration, it indirectly confirms our findings that fibrinolytics can work even beyond the traditional time limit of 7-10 d [8]. Unfortunately, more careful analysis of the duration is hampered because the authors presented data using medians (in the tables) and means (in the text).

In this study, there were 40 (of 84) children having septations on ultrasonography, with almost equal distribution in the two groups. This provides an excellent opportunity for sub-group analysis, to study the efficacy of urokinase in children with and without septated empyema. Seven of the 84 children also had loculated empyema; it would be interesting to learn the outcomes in them separately. I look forward to these clarifications through the correspondence section of the Journal.

Where are we at present? The balance of evidence favours intrapleural fibrinolytic therapy in childhood empyema in Indian institutions where empyema is primarily managed in Medical units. Even in institutions where empyema is managed in/by surgical units, fibrinolytic therapy may be a safer, cheaper, logistically easier (hence preferable) option to surgical management at presentation $[10,11]$. Traditionally, streptokinase has been the fibrinolytic of choice; this study [9] shows that urokinase is also feasible.

Where do we go from here? Given that empyema and its management in the Indian setting are considerably different from many of the settings where evidence was generated (in terms of younger age of children, delayed presentation, frequent malnourished state, predominance of Staphylococcus aureus, limited access to high-quality surgical management, etc), it is imperative that a robust multi-centre study be undertaken, to address the following questions: (i) What should be the timing of intrapleural fibrinolytics, i.e., what is the outer time limit of its successful administration? (ii) How many doses are optimal? (iii) Can therapy be shortened if multiple doses are administered on the same day? (iv) What is the comparison between streptokinase vs. urokinase in terms of efficacy, safety, and cost? (v) How does fibrinolytic compare to (up-front) surgical management? (vi) Can we predict which children initiated on medical therapy will eventually require surgical management? Naturally this will require collaboration among Pediatricians, Radiologists, Pediatric surgeons, (and perhaps adult Physicians/Surgeons caring for children) to evolve common definitions, standardization of management protocols, uniform radiologic criteria \& terminology, outcome measures and follow-up protocols. Although this is a somewhat tall order, it is both necessary and timely.

\section{Compliance with Ethical Standards}

\section{Conflict of Interest None.}

\section{References}

1. Balfour-Lynn IM, Abrahamson E, Cohen G, et al. BTS guidelines for the management of pleural infection in children. Thorax. 2005;60:11-21.

2. Long AM, Smith-Williams J, Mayell S, Couriel J, Jones MO, Losty PD. 'Less may be best'-pediatric parapneumonic effusion and empyema management: lessons from a UK center. J Pediatr Surg. 2016;51:588-91.

3. The Royal Children's Hospital Melbourne. Clinical Practice Guidelines. Pleural effusion and empyema. Available at: https:// www.rch.org.au/clinicalguide/guideline index/Pleural Effusion and Empyema/. Accessed 5 October 2019.

4. Empyema Care Guidelines. Available at: https://www.choc.org/wp/ wp-content/uploads/careguidelines/EmpyemaCareGuideline.pdf. Accessed 5 October 2019.

5. Shen KR, Bribriesco A, Crabtree T, et al. The American Association for Thoracic Surgery consensus guidelines for the management of empyema. J Thorac Cardiovasc Surg. 2017;153: e129-46.

6. Bradley JS, Byington CL, Shah SS, et al. The management of community-acquired pneumonia in infants and children older than 3 months of age: clinical practice guidelines by the Pediatric Infectious Diseases Society and the Infectious Diseases Society of America. Clin Infect Dis. 2011;53:e25-76.

7. Singh M, Mathew JL, Chandra S, Katariya S, Kumar L. Randomized controlled trial of intrapleural streptokinase in empyema thoracis in children. Acta Paediatr. 2004;93:1443-5.

8. Mathew JL, Soni V, Singh M, et al. Intrapleural streptokinase is effective and safe for children with multi-loculated empyema regardless of the time from disease onset. Acta Paediatr. 2018;107: 2165-71.

9. Nandan D, Agarwal S, Bidhuri N, Shrivastava K, Nanda P, Lata S. Role of intrapleural urokinase in empyema thoracis. Indian $\mathrm{J}$ Pediatr. 2019. https://doi.org/10.1007/s12098-019-03060-w.

10. Mathew JL, Lodha R, Sharma S. VATS or urokinase for treatment of empyema? Indian Pediatr. 2015;52:57-60.

11. Griffith D, Boal M, Rogers T. Evolution of practice in the management of parapneumonic effusion and empyema in children. $\mathrm{J}$ Pediatr Surg. 2018;53:644-6.

Publisher's Note Springer Nature remains neutral with regard to jurisdictional claims in published maps and institutional affiliations. 